\title{
Presencia del "ají nativo supano" (Capsicum chinense Jacq.) en el valle de Supe, Perú
}

\author{
Presence of the "supano native chili pepper" (Capsicum chinense Jacq.) in the Supe \\ valley, Perú
}

\section{J. Aliaga ${ }^{1,3}$, E. Portalatino ${ }^{1}$, K. Obregón ${ }^{1}$, A. Rodríguez ${ }^{2} \&$ J. Jimenez ${ }^{2}$}

\begin{abstract}
Resumen
Objetivos: Determinar la presencia in situ del "ají nativo supano" en el valle de Supe. Metodología: Se realizó una búsqueda exhaustiva para registrar las áreas de siembra en el ámbito de la cuenca baja del valle de Supe, en dos campañas agrícolas consecutivas. Resultados: Se ubicaron ocho parcelas entre las coordenadas geográficas $10^{\circ} 47^{\prime} 34^{\prime \prime}$ y $10^{\circ} 48^{\prime} 42^{\prime \prime}$ LS y $77^{\circ} 41^{\prime} 25^{\prime \prime}$ y $77^{\circ} 42^{\prime} 48^{\prime \prime}$ LO de la cuenca baja del valle de Supe, con áreas promedio de $256 \mathrm{~m}^{2}$. Estas parcelas corresponden a sistemas asociados a otros cultivos, con manejo convencional. Conclusiones: Por las condiciones de cultivo en las que se encuentra el ají nativo, corre el riesgo de desaparecer y con ello, la pérdida de valioso material genético.
\end{abstract}

Palabras clave: Capsicum chinense, germoplasma nativo, erosión genética, agricultores conservacionistas

\begin{abstract}
Objectives: To determine the in situ presence of the "supano native chili pepper" in the Supe valley. Methodology: An exhaustive search and registration of the planting areas in the area of the lower basin of the Supe valley was carried out in two consecutive agricultural seasons. Results: Eight plots were located between the geographical coordinates $10^{\circ} 47^{\prime} 34^{\prime \prime}$ y $10^{\circ} 48^{\prime} 42^{\prime \prime}$ LS y $77^{\circ} 41^{\prime} 25^{\prime \prime}$ y $77^{\circ} 42^{\prime} 48^{\prime \prime}$ LO of the lower basin of the Supe valley, with average areas of $256 \mathrm{~m}^{2}$. These plots correspond to systems associated with other crops, with conventional management. Conclusion: Due to the cultivation conditions in which the native chili is found, it runs the risk of disappearing and with it, the loss of valuable genetic material.
\end{abstract}

Keywords: Capsicum chinense, germplasm, genetic erosion, conservationists farmers

\section{Introducción}

Todas las especies del género Capsicum son originarias de América (Moscone et al., 2006; Ibiza et al., 2012; Rojas et al., 2016) y están formadas por alrededor de 20 especies silvestres y 5 taxones domesticados: C. pubescens, $C$. baccatum, $C$. annuum, $C$. chinense y $C$. frutescens (International Board for Plant Genetic Resources, 1983; Hernández-Verdugo et al., 1999). En el Perú, existen al menos 11 especies, entre domesticadas y silvestres (Brack, 2015).
Los taxones domesticados ahora tienen una distribución mundial, pero en el momento en que Colón descubrió el Nuevo Mundo, su distribución era limitada. Así, el Capsicum annuum var. Annuum se encontraba en Mesoamérica; Capsicum baccatum var. Pendulum, restringido a zonas del oeste y al este de los Andes; Capsicum pubescens, especie andina localizada en zonas de mediana elevación (2000 - 2500 msnm); y Capsicum chinense y C. frutescens, ubicados en zonas amazónicas (Nuez et al., 1996).

En el Perú, los ajíes son cultivados desde

\footnotetext{
${ }^{1}$ Universidad Nacional de Barranca, Barranca, Perú.

${ }^{2}$ Universidad Nacional Agraria La Molina, Lima, Perú.

${ }^{3}$ Autor para correspondencia: jualica@hotmail.com
} 
el límite con el Ecuador hasta la frontera con Chile y Bolivia y desde las zonas más altas de la cordillera de los andes hasta el desierto costero (Universidad Nacional Agraria La Molina, 2012; Brack, 2015).

Tomando como base el conocimiento campesino, acumulado y conservado durante generaciones (Instituto Nacional de Investigación y Extensión Agraria, 2006), existen dos estrategias básicas para la conservación de germoplasma vegetal: la conservación "in situ" y la conservación "ex situ" (Nodarse et al., 1998, citado por García et al., 2007). En "in situ" las especies de ajíes pueden conservarse como cultivadas en grandes extensiones para su comercialización, o como áreas reducidas de siembra para autoconsumo (Ministerio del Ambiente, 2016). La conservación in situ ha demostrado las potencialidades de los huertos familiares como auxiliares a la estrategia de la conservación de los bancos de germoplasma.

Actualmente se puede notar que la diversidad de especies de ají se está reduciendo a nivel de Latinoamérica, y esta situación puede deberse a la pérdida de los bosques tropicales y subtropicales, o a la desaparición lenta de los sistemas de la agricultura tradicional debido a proyectos de modernización rural, y de la pérdida de entradas en los bancos de genes (Brack, 2015). La conservación in situ es el único método práctico actualmente disponible para conservar una gran variedad de ecosistemas, especies y genes actualmente vulnerables, amenazados o en peligro (Food and Agriculture Organization, 1989). Por otro lado, el cambio climático y el crecimiento poblacional también afectan el desarrollo y conservación de estas especies y variedades en condiciones in situ.

A partir de la distribución geográfica del género es posible elaborar mapas de patrones de diversidad (García et al., 2006), que permitan la conservación de estos materiales genéticos que pueden ser utilizados para fines comerciales, de autoconsumo o para fines de mejoramiento genético. En ese sentido, los recursos fitogenéticos relacionados con el género Capsicum, adquieren gran relevancia por el potencial genético que presentan y por ser la base para obtener variedades mejoradas para diversos fines (Moreno et al., 2011), por lo que el objetivo de este trabajo fue determinar la presencia del "ají nativo supano" en la cuenca baja del valle de Supe.

\section{Metodología}

La presente investigación se desarrolló en la cuenca baja del río que Supe, localizada entre las coordenadas geográficas $10^{\circ} 50$ ' $28^{\prime \prime}$ " LS, $77^{\circ} 43^{\prime} 02^{\prime \prime}$ LO con altitud $6 \mathrm{msnm}$ y $10^{\circ} 54^{\prime} 50^{\prime \prime} \mathrm{LS}, 7^{\circ} 27^{\prime} 57^{\prime \prime} \mathrm{LO}$ con $475 \mathrm{msnm}$, comprendiendo los sectores: San Nicolás, Caleta Vidal, Molinos, Pan de Azúcar, Yamahuaca, Caral y Las Minas (Figura 1), durante las campañas agrícolas 2015/2016 y 2016/2017.

Se recurrió a visitar la Comisión de Regantes del valle de Supe, con la finalidad de obtener la información necesaria acerca de la presencia del "ají nativo supano". Luego de ubicadas las áreas con presencia de dicho material, se

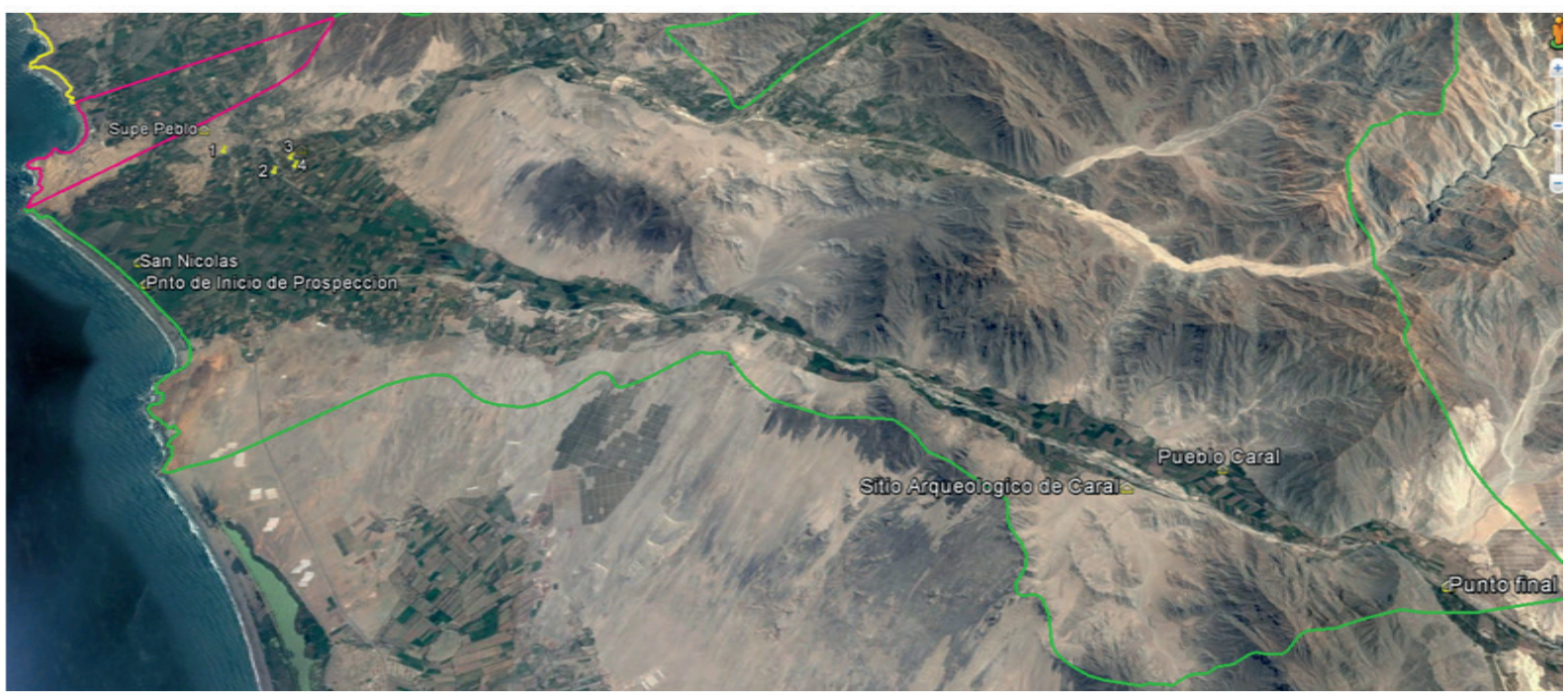

Figura 1. Prospección de áreas cultivadas del “ají nativo supano”. Campañas agrícolas 2015/2016 y 2016/2017. 
Tabla 1

Ubicación geográfica y áreas de siembra del "ají nativo supano" en el valle de Supe

\begin{tabular}{|c|c|c|c|c|c|}
\hline \multirow{2}{*}{ Sector } & \multirow{2}{*}{ Latitud S } & \multirow{2}{*}{ Longitud O } & \multirow{2}{*}{$\begin{array}{l}\text { Altitud } \\
\text { (msnm) }\end{array}$} & \multicolumn{2}{|c|}{ Área (m2)/Campaña } \\
\hline & & & & $2015 / 2016$ & $2016 / 2017$ \\
\hline \multirow{5}{*}{$\begin{array}{l}\text { La Campiña- } \\
\text { Supe }\end{array}$} & $10^{\circ} 48^{\prime} 24.56^{\prime \prime}$ & $77^{\circ} 41^{\prime} 47.71^{\prime \prime}$ & 69 & - & 200 \\
\hline & $10^{\circ} 48^{\prime} 32.36^{\prime \prime}$ & $77^{\circ} 41^{\prime} 49.44^{\prime \prime}$ & 51 & 300 & 300 \\
\hline & $10^{\circ} 48^{\prime} 42.75^{\prime \prime}$ & $77^{\circ} 41^{\prime} 25.42^{\prime \prime}$ & 52 & 100 & 100 \\
\hline & $10^{\circ} 48^{\prime} 38.72^{\prime \prime}$ & $77^{\circ} 41^{\prime} 55.58^{\prime \prime}$ & 48 & 400 & - \\
\hline & $10^{\circ} 48^{\prime} 33.36^{\prime \prime}$ & $77^{\circ} 41^{\prime} 51.08^{\prime \prime}$ & 62 & - & 300 \\
\hline \multirow{2}{*}{$\begin{array}{l}\text { La Campiña- } \\
\text { Río Seco }\end{array}$} & $10^{\circ} 48^{\prime} 1.20^{\prime \prime}$ & $77^{\circ} 41^{\prime} 35.15^{\prime \prime}$ & 58 & 500 & 300 \\
\hline & $10^{\circ} 48^{\prime} 13.52^{\prime \prime}$ & $77^{\circ} 41^{\prime} 28.78^{\prime \prime}$ & 58 & 270 & - \\
\hline Supe Pueblo & $10^{\circ} 47^{\prime} 34.67^{\prime \prime}$ & $77^{\circ} 42^{\prime} 48.30^{\prime \prime}$ & 68 & 150 & 150 \\
\hline
\end{tabular}

procedió a levantar información de áreas de siembra, de manejo del cultivo y de composición del agrosistema. Asimismo, estas unidades productivas fueron georeferenciadas con el uso de GPS (Garmin) y posteriormente se capturó la representación gráfica en Google Earth.

\section{Resultados y discusión}

Del total de área evaluada, se pudo observar que solamente ocho agricultores siembran el "ají nativo supano", con áreas promedio de las dos campañas de $256 \mathrm{~m}^{2}$, que pertenecen a los sectores de Supe Pueblo, La Campiña Río Seco y la Campiña de Supe, tal como se observa en la
Tabla 1. Estos sectores corresponden a altitudes comprendidas entre los 48 y $69 \mathrm{msnm}$. El "ají nativo supano" también es conocido como "ají campiñero" o "arnaucho campiñero".

De los ocho agricultores que siembran el cultivo, cuatro de ellos son los que sembraron consecutivamente y que se les reporta como agricultores guardianes del germoplasma in situ y ellos fueron: Raúl Quispe Villarreal, Alejandro Dávila Bueno, Aquiles Dávila Bueno y Alfredo Lara Enríquez (Figuras 2 y 3). Éste es un tipo de sistema de conservación de material genético referido por la Food and Agriculture Organization (2008), García et al., (2007) y Borges et al.,

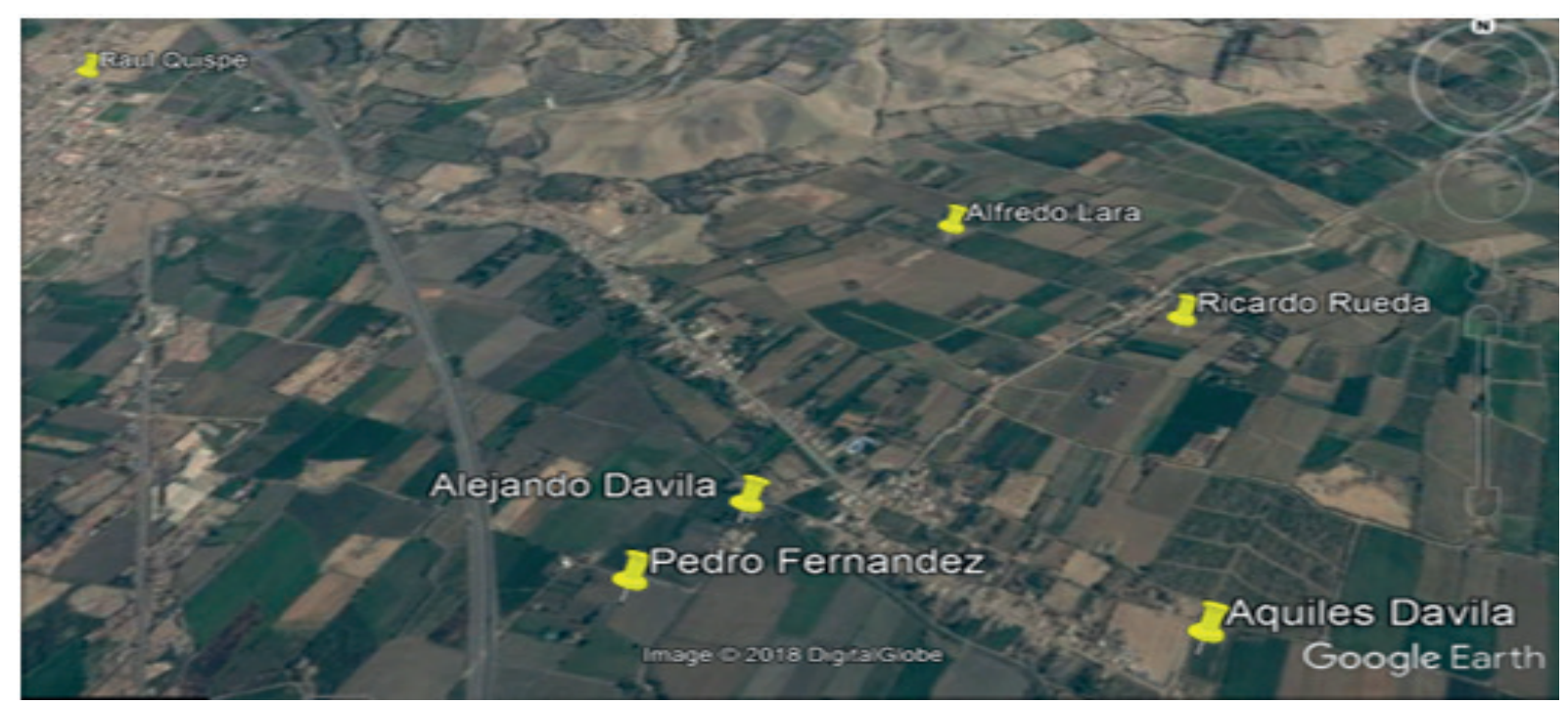

Figura 2. Mapa de distribución geográfica de las áreas cultivadas con “ají nativo supano” 2015/2016. 


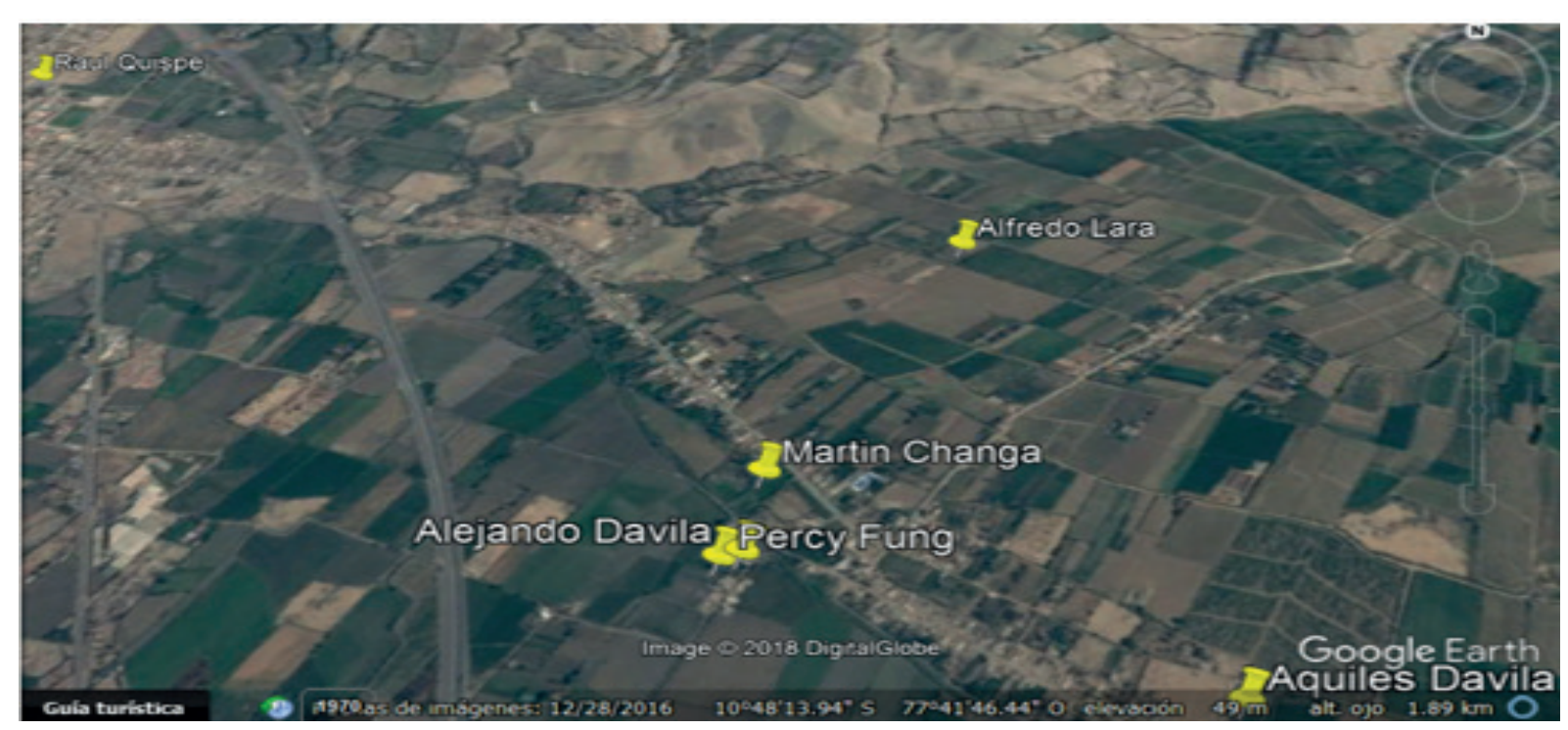

Figura 3. Mapa de distribución geográfica de las áreas cultivadas con “ají nativo supano" 2016/2017.

(2010). Estos agricultores se caracterizan por obtener sus propias semillas propias procedentes de la campaña anterior, previa selección y maduración en planta. La edad promedio de estos agricultores fue de 77 años.

Los sectores donde se observó el "ají nativo supano" pertenecen a una zona de vida de desierto pre montano, clima subtropical, que se caracteriza por presentar temperaturas medias entre $24.3{ }^{\circ} \mathrm{C}$ (verano) y $16.5{ }^{\circ} \mathrm{C}$ (invierno), con precipitaciones anuales inferiores a 25 $\mathrm{mm}$. Los suelos corresponden al tipo aluvial de profundos a superficiales, de textura media a moderadamente gruesa y grado de fertilidad baja (Flores, 2006). Las características antes descritas favorecen el desarrollo óptimo de los cultivos de ají (Ríos, 2017).

La producción del "ají nativo supano" es una actividad económica, practicada por los pequeños agricultores, y se caracteriza por formar parte de las siembras asociadas con el "ají escabeche" (Capsicum baccatum var. Pendulum), la yuca (Manihot esculenta Crantz) y el maíz chala (Zea mays L.). Las siembras se inician en el mes de julio, a través de almácigos en las mismas parcelas, y dos meses después se trasplantan en áreas adyacentes. Su manejo es convencional, con riegos por gravedad y uso frecuente de fertilizantes y plaguicidas, coincidiendo con lo reportado por Jäger et al (2013a) y Ríos (2017). Este cultivo, como otras especies de ajíes sembradas en los valles de la provincia de Barranca, es muy susceptible al ataque de una plaga conocida por los agricultores como "caracha" o "prodiplosis", cuyo nombre científico es Prodiplosis longifila Gadne, (Diptera: Cecidomyiidae), que para su control requiere de numerosas aplicaciones de insecticidas, llegando al extremo de abandonar su cultivo (Universidad Nacional Agraria La Molina, 2012; Martel, 2017).

La cosecha se inicia a partir del mes de noviembre y se prolonga hasta cinco meses después, correspondiendo a los meses de abril y mayo del año siguiente. Cada planta produce de 0.30 a $2.0 \mathrm{~kg}$, similar a los ajíes nativos de la especie $C$. chinense (Rojas et al., 2016). En el "ají nativo supano", como en otros ajíes, el crecimiento del fruto presenta tres fases: logarítmica, lineal y de senescencia (Bautista, 2017). En la fase logarítmica, donde el tamaño aumenta de forma exponencial a través del tiempo, el color es morado; en tanto que, en la fase lineal, donde el aumento del tamaño es continuo a una velocidad constante, el color se torna blanco amarillento, y finalmente en la fase de senescencia, donde la velocidad es decreciente del crecimiento a medida que el fruto alcanza la madurez el fruto, se torna anaranjado, para luego ser rojo al comienzo de la senectud. La demanda del mercado por este ají ocurre cuando el fruto presenta la coloración morada a crema, que es el estado en que el producto presenta mayor aroma, tal como lo ratifican Melgarejo et al. (2004) y Universidad Nacional Agraria La Molina (2012). 
Capsicum chinense Jacq. se reporta en la provincia de Barranca desde 1963, donde se cultivaban en huertos familiares para autoconsumo. Durante los años 1980, se empieza a cultivar en pequeñas parcelas con la intencionalidad de mercadeo y donde la distribución de semillas y almácigos ha sido de agricultor a agricultor, por familiaridad o vecindad, y que a la fecha continúa esa modalidad. Esto es similar a la propagación de los ajíes nativos, que realizan otros pequeños agricultores en el país, con la finalidad de conservar las semillas (Instituto Nacional de Investigación Agraria, 2007). Jäger et al. (2013a) mencionan que en la Costa norte (Norte Chico), este ají con la denominación de "Arnaucho", está reportado en la provincia de Barranca.

El "ají nativo supano" está presente en la culinaria local y regional en los platos típicos como el cebiche y el pato en ají (Jäger et al., 2013b), debido a la demanda continua durante todo el año, pero su abastecimiento es limitado en determinados meses del año, donde los precios se elevan, por lo que su conservación genética resulta de importancia socioeconómica y cultural para la localidad.

\section{Conclusiones}

En las campañas 2015/2016 y 2016/2017 se reportó la presencia del "ají nativo supano" entre altitudes de 48 a $69 \mathrm{msnm}$, perteneciente a los sectores de la Campiña de Supe, Campiña Río Seco y Supe Pueblo. Es cultivado por arraigo tradicional por pequeños agricultores quienes conservan el material genético in situ, con semillas propias. Por las condiciones en que se le encontró, puede estar en riesgo de erosión genética, recomendando promover el cultivo para su conservación.

\section{Agradecimientos}

Agradecimiento a la Universidad Nacional de Barranca, que financió el estudio a través del proyecto "Caracterización y sostenibilidad del cultivo de Capsicum chinense Jacq. "ají nativo Supano" en la Cuenca baja del río Supe, Barranca" fuente de financiamiento de Donaciones y Transferencias, Resolución $\mathrm{N}^{\circ}$ 206-2015-CO-UNAB.

\section{Referencias}

Bautista, M. (2017). Determinación del momento de cosecha de seis accesiones de aji (Capsicum spp.) nativos (tesis de pregrado). Universidad Nacional Agraria La Molina, Lima, Perú.

Borges, L., Cervantes, L., Ruiz, J., Soria, M., Eyes, V. \& Villanueva, E. (2010). Capsaicinoides en chile habanero (Capsicum chinense jacq.) bajo diferentes condiciones de humedad y nutrición. Terra Latinoamericana, 28(1), 35-41.

Brack, A. (2015). Catorce recursos genéticos que cambiaron al mundo y uno que lo cambiara. 1ra Edición. Lima, Perú: Gráfica Biblos S.A.

Food and Agriculture Organization (2008). Agricultura para la biodiversidad. Roma. Disponible en: http://www.fao.org/tempref/ docrep/fao/010/i0112s/i0112s.pdf

Food and Agriculture Organization (1989). Plant Genetic Resources: Their Conservation in situ for Human Use. Food and Agriculture Organization of the United Nations (FAO), Roma, Italia. Disponible en: http://www. fao.org/3/i1500e/i1500e brief.pdf

Flores, L. (2006). Estudio de las unidades residenciales en el subsector 12 de Caral, Valle de Supe-Perú (tesis de pregrado). Universidad Nacional Mayor de San Marcos, Lima, Perú.

García, M., Baena, D., Vallejo, F. (2006). Estudio de la diversidad genética de las accesiones de Capsicum spp. del banco de germoplasma de la Universidad Nacional de ColombiaSede Palmira (tesis doctoral). Universidad Nacional de Colombia, Palmira, Colombia.

García, L., De Feria, M. \& Acosta, K. (2007). Aspectos básicos de la conservación in vitro de germoplasma vegetal. Biotecnología Vegetal, 7(2), $67-79$.

Hernández-Verdugo, S., Aranda-Dávila, P. \& Oyama, K. (1999). Síntesis del conocimiento taxonómico, origen y domesticación del género Capsicum. Review of taxonomy, origin and domestication of the genus Capsicum. Boletín de la Sociedad Botánica de México, 64, 65-84.

Ibiza, V., Joaquín, J. \& Nuez, F. (2012). Taxonomy and genetic diversity of domesticated Capsicum species in the Andean region. 
Genetic Resources and Crop Evolution, 59(6), 1077-1088.

International Board for Plant Genetic Resources.

(1983). Genetic resources of capsicum: A global plan of action. Rome, Italy: IBPGR Secretariat.

Instituto Nacional de Investigación Agraria (2007). Los Cultivos Nativos en las Comunidades del Perú. Proyecto Perú. Conservación in situ de los cultivos nativos $y$ sus parientes silvestres. Lima, Perú: Promotora Lima Ediciones.

Instituto Nacional de Investigación y Extensión Agraria (2006). Manual para la caracterización in situ para los cultivos nativos. Lima, Perú: Ministerio de Agricultura.

Jäger, M., Jiménez, A. \& Amaya, K. (2013a). Guía de oportunidades de mercado para los ajíes nativos de Perú. Disponible en: https://www.bioversityinternational.org/ fileadmin/_migrated/uploads/tx_news/ Guia_de_oportunidades_de_mercado_ para_los_ajies_nativos_de_Peru_1729.pdf

Jäger, M., Jiménez, A. \& Amaya, K. (2013b). Las cadenas de valor de los ajies nativos de Perú. Disponible en: https://www. bioversityinternational.org/fileadmin/_ migrated/uploads/tx_news/Las_cadenas _ de_valor_de_los_aj\% $3 \%$ ADes_nativos de_Peru_1730.pdf

Martel, Y. (2017). Eficiencia del Galil 300 SC (Imidacloprid $250 \mathrm{~g} / \mathrm{l}$. + Bifetrin $50 \mathrm{~g} / \mathrm{l}$.) en el control de Prodiplosis longifila, en el cultivo de pimiento (Capsicum annuum L.) bajo las condiciones edafoclimáticas del distrito de Barranca, provincia Barranca (tesis de pregrado). Universidad Nacional Santiago Antúnez de Mayolo, Huaraz, Perú.

Melgarejo, L., Hernández, M., Alberto, J. \& Bardales, X. (2004). Caracterización y usos potenciales del banco de germoplasma de aji amazónico. Bogotá, Colombia: Universidad Nacional de Colombia.
Ministerio del Ambiente (2016). Determinación de la distribución de la diversidad genética y estudio socioeconómico del ají. Informe final. Ministerio del Ambiente (MINAM). Disponible en: http://genesperu.minam. gob.pe/wp-content/uploads/2016/09/Aji1Determinaci\%C3\%B3n-de-la-distribucionde-la-diversidad-genetica-y-estudiosocioeconomico-del-aji.pdf

Moreno, C., Avendaño, C., Mora, R., Cadena, J., Aguilar, V. \& Aguirre, J. (2011). Diversidad morfológica en colectas de chile guajillo (Capsicum annuum L.) del centro-norte de México. Revista Chapingo, 17, 23-30

Moscone, E. A., Scaldaferro, M. A., Grabiele, M., Cecchini, N. M., Sánchez García, Y., Jarret, R., Daviña, J., Ducasse, A., Barboza, G. \& Ehrendorfer, F. (2006). The evolution of chili peppers (Capsicum-Solanaceae): a cytogenetic perspective. Acta horticulturae, 745, 137-170. doi.org/10.17660/ ActaHortic.2007.745.5

Nuez, F., Gil, R. \& Costa, J. (1996). Cultivo de pimientos, chiles y ajies. Madrid, España: Mundi Prensa.

Ríos, M. (2017). Caracterización agro morfológica de diez selecciones de aji escabeche (Capsicum baccatum var. pendulum), bajo condiciones de La Molina (tesis de pregrado). Universidad Nacional Agraria La Molina, Lima, Perú.

Rojas, R., Patel, K., Ruiz, K., Calderón, R., Asencios, E., Quispe, F. \& Marcelo, M. (2016). Ajíes nativos peruanos. Caracterización agro-morfológica, químico-nutricional y sensorial. Lima, Perú: Universidad Peruana Cayetano Heredia.

Universidad Nacional Agraria La Molina (2012). El punto de aji. Investigaciones en Capsicum nativos. Lima, Perú: Programa de Hortalizas-Universidad Nacional Agraria La Molina. 